\title{
Agent-based simulation in a normative environment using the EPMP domain.
}

\begin{abstract}
Simulation is a way of doing thought experiments besides the deduction and induction methods. Agent-based Simulation (ABS) falls under the domain of artificial intelligence when agent is used to perform certain tasks such as behaviour prediction, optimization of functions or time-constrained work-flow management. This work discussed the use of ABS on a computational normative framework based on a set of empirical characteristics that influence agents' performance in time-constrained environment. The ABS simulates a domain called the Examination Preparation and Moderation Process (EPMP) which entails document submission processes with deadlines. The simulation is conducted in six different environments and the results of the agent performance in each environment are presented and discussed. The results indicate that the simulation conducted in the EPMP is suitable and effective for evaluating normative agent-based systems.
\end{abstract}

Keyword: Agent-based simulation; EPMP; Normative framework; Software agent; Timeconstrained environment. 